(1) the main affect for trial blocks (F $=15.35 . \mathrm{df}=2 / 80 . \mathrm{p}<.01)$. which was dice (1) the increase in performance across trials. And (2) the Trial Blocks by Training Croups interaction $(F=6.48 . \mathrm{df}=2 / 80$. $\mathrm{p}<.01$ ). Follow-up tests indicated that the difference between the two groups for the first block of trials was nonsignificant $(t=.53 . d f=42, p>.20)$. while Group $T$ made significantly more correct responses than Group XT for both the second and third trial blocks $(t=3.71$ and 9.35 . respectively, $d f=42$ and $p<.01$ in each case). While inspection of Table 1 reveals an apparent interactive effect of training and age. this interaction was not statistically significant $(\mathrm{F}=1.45$. $\mathrm{d} f=1 / 40 . \mathrm{p}>.20$ ).

Fifteen $S s$ in Group T. but only six Ss in Group NT. reached criterion. A chi-square analysis indicated this difterence to be significant $\left(\chi^{2}=7.38 . \quad \mathrm{df}=1, \mathrm{p}<.01\right)$. Somewhat surprising. however, was the finding that only 6 of the 15 children in Group $T$ reaching criterion were able to verbalize the solution of the oddity problem. Thus. most of these children were able to note stimulus similarities and differences during the training phase and were, in addition, able to do this in the test phase in that they attained the performance criterion but, nonetheless, they were unable to overtly verbalize the principle involved in the problem solution. of the six $\mathrm{Ss}$ in Group NT reaching criterion. four were able to verbalize the problem solution. The youngest child in this group to reach criterion was 51 months of age.

The results of this study provide some evidence supporting the hypothesis of Lipsitt \& Serunian (1963) that reading-readiness training may accelerate a child's acquisition of the oddity solution. In this regard it should be noted that the duration of the training phase was only $10 \mathrm{~min}$, the training was quite informal, and that the primary emphasis during this phase was upon instructing the child to attend to dimensional similarities and differences (size and form) not involved in the oddity problem.

\section{REFERENCES}

ELLIS. N. R.. \& SLOAN. W. Oddity learning as a function of mental age. Journal of Comparative \& Physiological Psychology, 1959, 52, 228-230.

LINDQUIST, E. F. Design and analysis of experiments in psychology and education. New York: Houghton Mifflin, 1953.

LIPSITT. L. P., \& SERLNIAN, S. A. Oddity problem learning in young children. Child Development. 1963, 34. 201-206.

PENN. I. E., SINDBERG, R. M.. \& WOHLHLETER, M. J. The oddity concept in severely retarded children. Child Development, $1969,40,153-161$

\title{
Anticipatory fear and judgments of shock intensity
}

\author{
LOUIS R. FRANZINI, San Diego State \\ College, San Diego, Calif. 92115
}

Three levels of shock intensity, individually calibrated for each of 30 male Ss, and four interstimulus intervals $(5,10$, 30. and $75 \mathrm{sec}$ ) were presented randomly in all combinations four times. Half the trials were data trials, i.e., terminated with a shock. Ss' judgments of shock intensity remained unaffected by increases in the interval or by the development of a hypothetical emotional response within $S$ while he awaited possible shock.

It seems reasonable that affective anticipatory fear, may develop within Ss during the temporal interval between a warning signal and the probable occurrence of a noxious stimulus. Franzini (in press) has applied the magnitude-estimation technique to assess directly the averseness of that period during which there is a high probability of the male college student S's receiving a noxious electric shock. He found the degree of perceived averseness to be a function of the interaction of the level of shock anticipated and the length of the interval spent awaiting the shock. Magnitude estimations of the averseness of the interval were linearly related to the size of the interval prior to shock delivery at all levels of anticipated shock.

Thus. Franzini's data indicate that the period awaiting possible shock is aversive. Any fear or anxiety that might develop in the interval following a signal that shock may follow could be conceived of as an additional drive source. Increased drive level would be expected to augment one's perception of the intensity of a noxious stimulus. This study is designed to assess the hypothesis that as the interval between warning signal and anticipated shock delivery increases, magnitude estimations of shock intensity increase.

\section{METHOD}

Thirty male Ss were recruited from undergraduate psychology classes. Shock was delivered through electrodes strapped to Ss' nonpreferred forearm. The circuit was designed to function as a constant-current system. A 336,000-ohm resistor was placed in series with the shock output so that interindividual differences in skin resistance would not significantly alter the actual delivered current, which ranged from 0.10 to $6.1 \mathrm{~mA}$. Shock intensity was regulated by a Variac. Shock duration was constant at $0.75 \mathrm{sec}$ in all conditions.

The independent variables were three levels of shock intensity, individually calibrated for each $\mathbf{S}$ according to his range of tolerance, and four levels of intratrial interval $(5,10,30$, and $75 \mathrm{sec})$. These levels were chosen to conform with the paradigm of Franzini (in press) in which different Ss judged the averseness of the interval preceding shock. It was assumed that the action of higher-order symbolic processes in human Ss would serve to maintain or even augment any emotional response that might build following CS onset. Each $S$ made 24 judgments, having been presented randomly each of the 12 conditions twice. A trial consisted of a light of $1-\sec$ duration (CS), the prescribed time interval, and the termination which would be a shock (UCS) of the prescribed intensity or a single click. Trials ending with a click were nondata trials and 


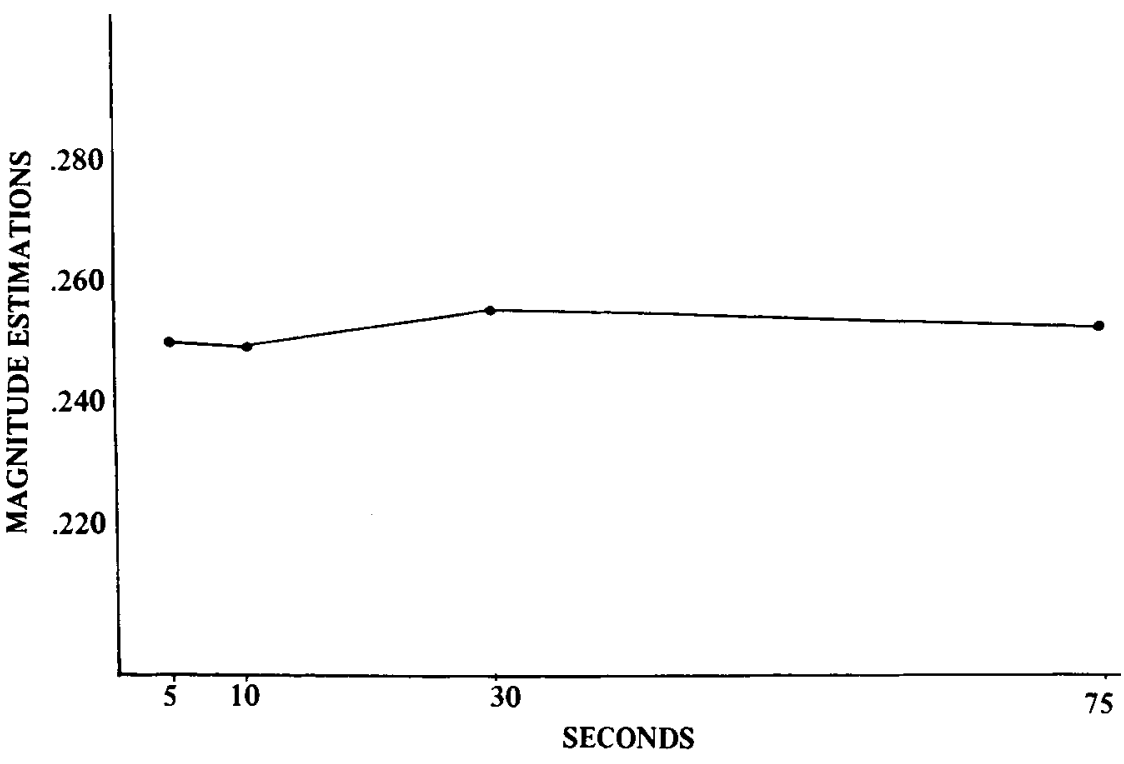

constituted half of the total of 48 trials. Trial orders and the levels prescribed for shock intensity and size of interval were presented randomly. A modulus, given the value of 50 by $E$, consisted of a shock of medium intensity level for each particular $S$ after an interval of $30 \mathrm{sec}$.

\section{PROCEDURE}

The threshold and tolerance level for each $S$ were determined in a preliminary scaling procedure that had a twofold purpose: (1) to give $\mathrm{Ss}$ some experience with the shock stimuli prior to the actual collection of magnitude estimation data and (2) to permit $E$ to adjust the actual current intensities delivered according to the range of tolerable sensation applicable to the $\mathbf{S}$.

Two intensity values at each of the three shock levels (low, medium, and high) were calculated accoring to ratio formulae derived from scaling data of pilot Ss. In general, the extremes of the current intensities can be described as the lower value in the low intensity range being slightly above the minimally perceptible intensity to allow for accommodation, while the higher value at the high intensity level was simply the S's maximally tolerable value. Magnitude estimation data from the two data trials were averaged for each level.

Ss were told that the flashing of the light signified the beginning of a trial and that $50 \%$ of the trials would terminate with a shock and $50 \%$ with a click only. They would not know beforehand whether a given trial would end with a shock or not or what level of shock might be expected on that trial should a shock be prescribed. Instructions were read to the Ss to make their estimates of the strength of the shocks in proportion to the reference shock which had been assigned the value of 50. Several verbal examples were described but without actual demonstration of shock.

RESULTS
Magnitude estimation data were transformed to proportions for each $\mathrm{S}$ individually to correct for the possibility of any Ss using unusual ranges of numbers for their judgments. The statistical analyses were performed on the transformed data. The method of orthogonal polynomials was used to obtain the component trends of the treatment and residual parts of the analysis of variance (Grant, 1956).

The hypothesis, that as the interval between CS and UCS increases magnitude estimations of shock (UCS) intensity will increase, was not supported. As the interstimulus interval was increased across the four levels from 5 to $75 \mathrm{sec}$, Ss' means of the proportional magnitude estimations remained essentially unaffected. Figure 1 shows the plot of the mean proportional magnitude estimations pooled over all shock levels as a function of CS-UCS interval. The curve has a slope of near zero.

The analysis of variance of trends of the mean proportional magnitude estimations of shock intensity was computed separately for low, medium, and high shock levels and for all shock levels pooled. In none of the analyses did the main treatment effect, manipulation of the CS-UCS interval, or its linear, quadratic, or cubic components reach statistical significance. Analyses of variance of magnitude estimations as a function of increasing shock levels at each level of CS-UCS interval yielded highly significant $F$ values, indicating that $S$ s had readily discriminated the three levels of shock as intended.
Fig. 1. Means of pooled proportional magnitude estimations of shock intensity as a function of interstimulus interval.

\section{DISCUSSION}

Contrary to expectation, the Ss of this study showed a remarkable consistency in their ability to give magnitude estimations of shock intensity without influence from a variable waiting period before shock delivery or from any presumed internal emotional responses (fear or anxiety) that could have accrued differentially during that period. It was of little consequence whether the shock was one of low, medium, or high intensity or whether it came after a very brief $(5-\mathrm{sec})$ or a relatively long $(75-\mathrm{sec})$ waiting period. Although Hill et al (1952) claimed to have obtained a distortion in Ss' perception of shock intensity as a function of their augmented anxiety level, the judgments of the Ss of the present study were not similarly affected.

Using a similar paradigm, Franzini (in press) reported that $S s$ do experience increased averseness of the interval prior to anticipated shock. It had been predicted that a buildup of anxiety within an $S$ in the present study would function as an additive drive and serve to inflate the overt response of his magnitude estimations. It remains possible that the mechanism did operate in this way but that the expected increment in the overt response occurred in the form of increased auditory intensity and motoric forcefulness of the Ss' verbal behavior. No attempts to obtain such measures were made in this study. No reasonable test of the preceding theoretical mechanism per se could be made without these more appropriate measures of response magnitude.

\section{REFERENCES}

BROWN, J., KALISH, H., \& FARBER, I. Conditioned fear as revealed by magnitude of startle response to an auditory stimulus. Journal of Experimental Psychology, 1951, 41, 317-328.

FRANZINI, L. Magnitude estimations of the averseness of the interval preceding shock. Journal of Experimental Psychology, in press.

GRANT, D. Analysis-of-variance tests in the analysis and comparison of curves. Psychological Bulletin, 1956, 53, 141-152.

HILL, H., KORNETSKY, C., FLANARY, H., \& WIKLER, A. Effects of anxiety and morphine on discrimination of intensities of painful stimuli. Journal of Clinical Investigation, 1952, 31, 473-480.

SIDMAN, M., \& BOREN, J. The relative aversiveness of warning signal and shock in an avoidance situation. Journal of Abnormal \& Social Psychology, 1957, 55, 339-344. NOTE

1. The data reported here were gathered as part of a PhD dissertation submitted to the graduate faculty of the University of Pittsburgh This paper was prepared while the author was at San Diego State College. 